\title{
Investigation of the flux of albedo muons with NEVOD-DECOR experimental complex
}

\author{
S.S. Khokhlov*, D.V. Chernov, A.N. Dmitrieva, V.A. Khomyakov, V.V. Kindin, \\ R.P. Kokoulin, K.G. Kompaniets, V.S. Kruglikova, V.V. Shutenko, I.I. Yashin \\ National Research Nuclear University MEPhI (Moscow Engineering Physics Institute), Moscow \\ 115409, Russia \\ E-mail: sskhokhlov@mephi.ru
}

\begin{abstract}
Results of investigations of the near-horizontal muons in the range of zenith angles of 85-95 degrees are presented. In this range, so-called 'albedo' muons (atmospheric muons scattered in the ground into the upper hemisphere) are detected. Experimental data of two series of measurements obtained with the NEVOD-DECOR experimental complex with the duration of about 20 thousand hours 'live' time are analyzed. Monte Carlo simulations of multiple scattering of muons in the ground with a flat surface are performed with different models. The comparison of experimental data and simulation results shows that the main contribution to the flux of albedo muons in the range of angles $\theta=91^{\circ}-94^{\circ}$ is given by the multiple Coulomb scattering.
\end{abstract}

The 34th International Cosmic Ray Conference,

30 July - 6 August, 2015

The Hague, The Netherlands

* Speaker. 


\section{Introduction}

Investigating the near-horizontal flux of atmospheric muons is of interest both in the context of studying high-energy cosmic rays and of estimating the background in neutrino experiments. Modern neutrino observatories are deployed in lakes, seas and ices of Antarctica (BNT-200+, ANTARES, IceCube) and reach an effective volume of 1 cubic $\mathrm{km}$. Their operation is based on the detection of Cherenkov radiation emitted by relativistic charged particles passing through the water (ice) of detector. As a result of interaction with the target nuclei, neutrinos passing through the Earth can generate a muon or a cascade which comes from the lower hemisphere and is registered by the detection systems. However, the muons coming from the upper hemisphere may scatter in the soil or water (ice) and thus come from the lower hemisphere, these are so-called albedo muons. Such muons are one of the main sources of non-apparatus background in shallow depth neutrino experiments. The theoretical estimations of albedo flux of muons depend on scattering models which give different results, and therefore the experimental data on atmospheric muons from the lower hemisphere are important for neutrino physics.

Experimental data on albedo muon flux are rather scarce. First studies of albedo muons with NEVOD-DECOR detectors were conducted in 2002-2004 [1, 2]. In the present paper, new results of albedo muon investigations with considerably enhanced detection system of the Cherenkov water detector NEVOD are presented.

\section{Analysis of experimental data}

The experimental complex NEVOD-DECOR is dedicated to investigations of all components of cosmic rays on the Earth's surface, including albedo muons. The complex is based on the NEVOD Cherenkov water detector (CWD) [3] which detecting system has the form of a spatial lattice with 91 quasispherical modules (QSMs). Each QSM contains six photomultipliers with flat photocathodes directed along orthogonal axes. This construction allows us to determine the direction of the Cherenkov radiation at the level of one QSM. The coordinate detector DECOR [4] is deployed around the NEVOD. DECOR includes eight supermodules (SMs) which consist of eight vertically suspended planes of plastic streamer tube chambers with resistive cathode coating with the total sensitive area $70 \mathrm{~m}^{2}$. Chamber planes are equipped with two-coordinate external strip readout system. Detector DECOR allows to localize tracks of near-horizontal muons with high angular (better than 1 degree) and spatial (about $1 \mathrm{~cm}$ ) accuracy and also allows to determine the muon direction by means of time-of-flight technique.

Experimental data of two series are used: $10^{\text {th }}$ series which was conducted from December 2011 to March 2013 (7945 hours 'live' time), and $11^{\text {th }}$ series for the period from July 2013 to April 2015 (11897 hours 'live' time) performed after the startup of a new water purification system [5]. Events with two triggered SMs in opposite short galleries (SM00, SM01 and SM06, SM07), with tracks (presumably of one muon) reconstructed by each supermodule and coinciding within a cone of $5^{\circ}$ are selected. Then the midpoints of tracks within each SM are connected by a line, which represents the 'average' muon track with estimation of zenith angle $\theta_{\text {OT }}$ (see figure 1 ). The events in which the muon passes closer than $3 \mathrm{~cm}$ from the boundary of SM are rejected in order to decrease the edge effects. 


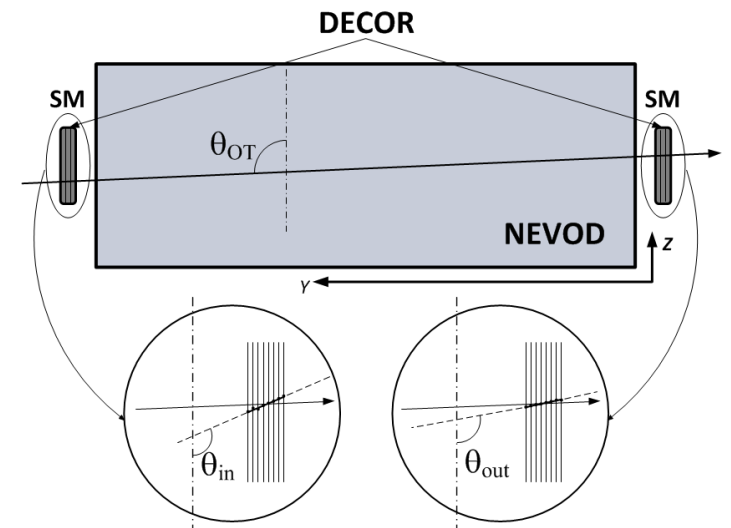

Figure 1: Scheme of the event with a near-horizontal muon. Definition of basic angles.

Such events are registered in the zenith angle range of $85^{\circ}-95^{\circ}$ and are referred to as 'OneTrack' events. The threshold energy of muons for such events is about $7 \mathrm{GeV}$. An example of reconstructed 'OneTrack' event is shown in figure 2. Tracks that are reconstructed according to each supermodule are shown in blue, the average reconstructed track is shown in green. Triggered QSMs are marked by crosses, and triggered PMTs by circles.

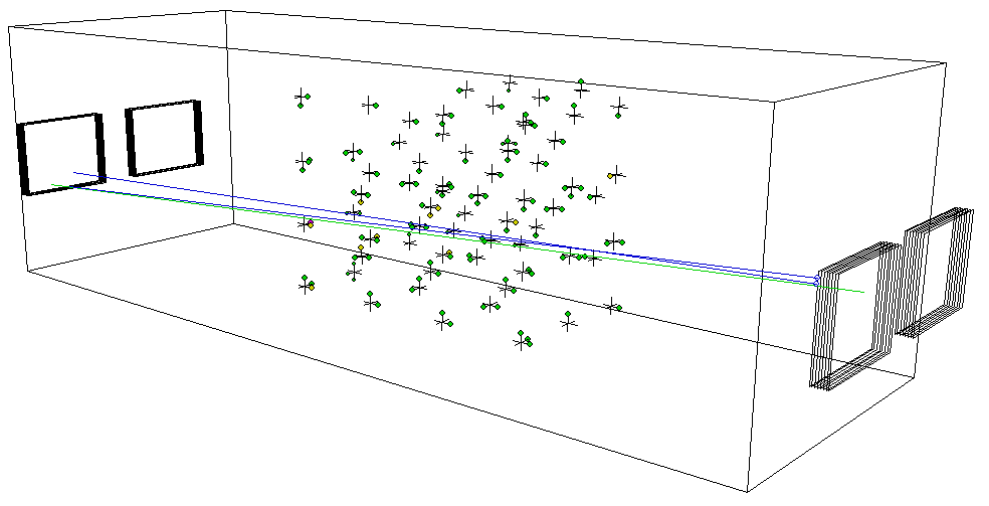

Figure 2: Example of reconstructed 'OneTrack' event.

Since the muon flux from the upper hemisphere is several orders of magnitude greater than the flux moving upward (from below the horizon), a key element in detecting albedo muons (scattered in the upper layers of the ground) is reliable determination of the particles' motion direction. Two methods for determining the direction can be used in the NEVOD-DECOR experimental complex simultaneously: the time-of-flight technique and measuring of Cherenkov radiation of charged particles in water.

Normally, most of the hit photomultipliers are oriented toward the direction of muon arrival, so the direction of particle motion can be determined from the difference between the numbers of oppositely-oriented triggered photomultipliers. For selected events, it is convenient to use photomultipliers directed toward SM00, SM01 (no. 3 PMTs in each QSM) and SM06, SM07 (no. 1 PMTs, respectively) of the coordinate-tracking detector. The relative difference between total amplitudes $\delta A$, which considers not only PMT actuation but the magnitude of a response as well, is actually more informative: 


$$
\delta A=\frac{\Sigma A_{3}-\Sigma A_{1}}{\Sigma A_{3}+\Sigma A_{1}}
$$

here

$\Sigma A_{3}$ is the total amplitude of the no. 3 PMTs directed toward SM00 and SM01;

$\Sigma A_{1}$ is the total amplitude of the no. 1 PMTs directed toward SM06 and SM07.

Figure 3 (a) presents the detailed distribution of the relative difference between the total amplitudes $\delta A$ for 'OneTrack' events. The right peak corresponds to muons coming from SM00, SM01 to SM06, SM07, while the left peak corresponds to the opposite direction. As can be seen from the figure, the peaks registered in the $11^{\text {th }}$ series are resolved better than in the $10^{\text {th }}$ series due to the increased water transparency after turning on a new water purification system.

The triggering system of the coordinate-tracking detector provides information on the relative times of triggered supermodule actuation with an increment of $25 \mathrm{~ns}$. The time of muon transit between SMs is about $90 \mathrm{~ns}$, so the direction can be identified using the time-of-flight technique as well. The distribution of time differences between signals from SMs located in opposite short galleries is shown in figure 3 (b).
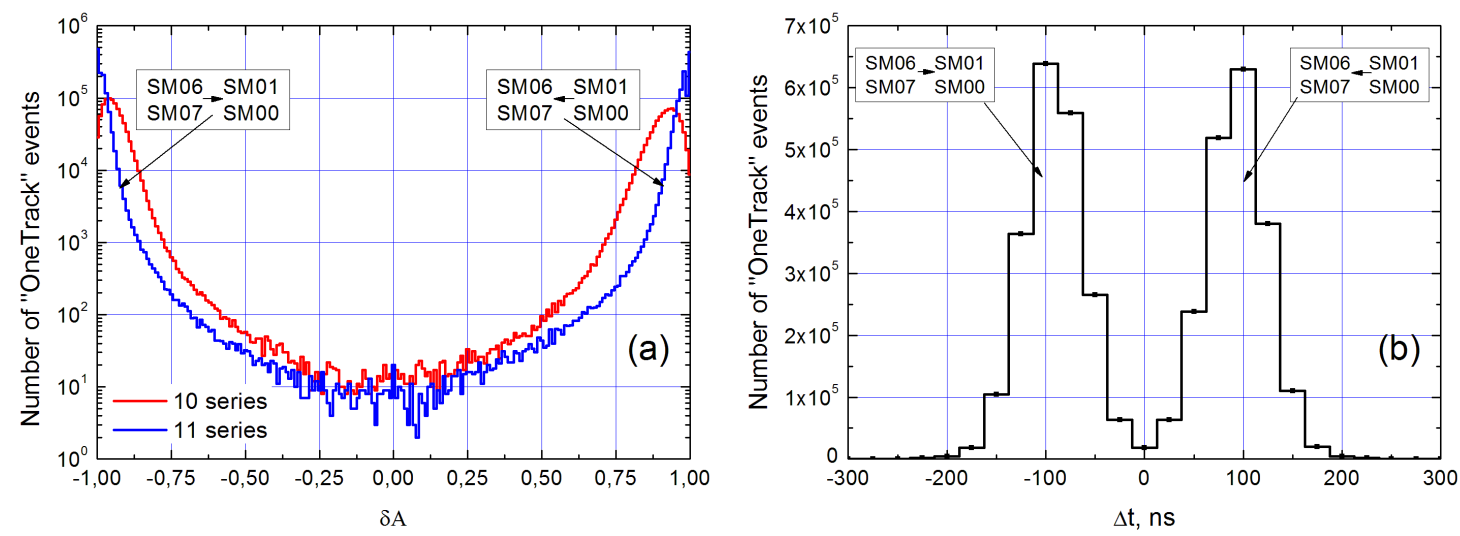

Figure 3: (a) Distribution of 'OneTrack' events according to the relative difference between the total amplitudes of opposite PMTs for $10^{\text {th }}$ series (red line) and $11^{\text {th }}$ series (blue line); (b) distribution of 'OneTrack' events according to the time difference between signals from SMs (total for $10^{\text {th }}$ and $11^{\text {th }}$ series of measurements).

\subsection{Criteria for determining the direction of the muon}

Amplitude and time-of-flight techniques are independent, therefore we can estimate the efficiency and the upper limit of the error of the criterion for the relative difference between the total amplitudes of PMTs by imposing rigid conditions for the time difference $|\Delta t|$. To accomplish this, we select events with $|\Delta t| \geqslant \Delta t_{c r}$ for which the error rate in determining direction is a priori low; events with zenith angles $89^{\circ}-91^{\circ}$ are excluded from this consideration. Events exceeding a given threshold $\delta A_{c r}$ are then selected among them. If the data on direction found by using the parameters $|\Delta t|$ and $\delta A$ contradicted each other, it was decided that the direction based on the relative difference between the amplitudes was false. The selection was carried out for various values of $|\Delta t|$ and $\delta A_{c r}$. Table 1 shows the estimates of the efficiency of selection and the error of amplitude technique of determining direction for two series. 


\begin{tabular}{|c|c|c|c|c|}
\hline \multicolumn{3}{|c|}{10 series: selection } & \multicolumn{2}{c|}{11 series: selection } \\
$\left(\theta \leqslant 89^{\circ}\right.$ and $\theta \geqslant 91^{\circ}$, and $\left.|d t| \geqslant 50 \mathrm{~ns}\right)$ & $\left(\theta \leqslant 89^{\circ}\right.$ and $\theta \geqslant 91^{\circ}$, and $\left.|d t| \geqslant 50 \mathrm{~ns}\right)$ \\
\hline$|\delta A|$ & $\begin{array}{c}\text { Efficiency of } \\
\text { identification, } \%\end{array}$ & Error rate & Efficiency of & Error rate \\
\hline$\geqslant 0.6$ & $99.726 \pm 0.005$ & $(2.78 \pm 0.47) \times 10^{-5}$ & $99.899 \pm 0.003$ & $(0.91 \pm 0.25) \times 10^{-5}$ \\
\hline$\geqslant 0.7$ & $99.386 \pm 0.007$ & $(1.68 \pm 0.37) \times 10^{-5}$ & $99.821 \pm 0.004$ & $(0.63 \pm 0.21) \times 10^{-5}$ \\
\hline$\geqslant 0.8$ & $97.410 \pm 0.014$ & $(1.38 \pm 0.34) \times 10^{-5}$ & $99.622 \pm 0.005$ & $(0.35 \pm 0.16) \times 10^{-5}$ \\
\hline
\end{tabular}

Table 1: Estimated efficiency and error rate of the amplitude technique.

The time-of-flight technique is analyzed in a similar manner by preselecting events based on the relative difference $|\delta A|$ between the amplitudes. The results are given in Table 2.

\begin{tabular}{|c|c|c|c|c|}
\hline \multicolumn{3}{|c|}{10 series: selection } & \multicolumn{2}{c|}{11 series: selection } \\
\multicolumn{2}{|c|}{$\left(\theta \leqslant 89^{\circ}\right.$ and $\theta \geqslant 91^{\circ}$, and $\left.|\delta A| \geqslant 0.7\right)$} & $\left(\theta \leqslant 89^{\circ}\right.$ and $\theta \geqslant 91^{\circ}$, and $\left.|\delta A| \geqslant 0.7\right)$ \\
\hline$|\Delta t|$, ns & $\begin{array}{c}\text { Efficiency of } \\
\text { identification, } \%\end{array}$ & Error rate & Efficiency of & Error rate \\
\hline$\geqslant 25$ & $99.27 \pm 0.01$ & $(2.66 \pm 0.05) \times 10^{-3}$ & $99.367 \pm 0.007$ & $(2.01 \pm 0.04) \times 10^{-3}$ \\
\hline$\geqslant 50$ & $96.27 \pm 0.02$ & $(1.27 \pm 0.03) \times 10^{-3}$ & $96.488 \pm 0.015$ & $(0.88 \pm 0.02) \times 10^{-3}$ \\
\hline$\geqslant 75$ & $83.92 \pm 0.03$ & $(0.53 \pm 0.02) \times 10^{-3}$ & $84.10 \pm 0.03$ & $(0.32 \pm 0.02) \times 10^{-3}$ \\
\hline
\end{tabular}

Table 2: Estimated efficiency and error rate of the time-of-flight technique.

The final criteria are selected on the basis of the condition that there must be a low ratio of contradictory events while a high efficiency of identification is maintained ( $\delta A \geqslant 0.7$ and $\Delta t \geqslant 50 \mathrm{~ns}$ or $\delta A \leqslant-0.7$ and $\Delta t \leqslant-50 \mathrm{~ns}$ ). When amplitude and time-of-flight techniques are used jointly, the efficiency and the rejection factor are equal to:

$$
\begin{array}{ll}
\eta=\eta_{\delta A} \cdot \eta_{\Delta t}=95.68 \% & R=R_{\delta A} \cdot R_{\Delta t}=2.13 \times 10^{-8} \text { (for the } 10^{\text {th }} \text { series); } \\
\eta=\eta_{\delta A} \cdot \eta_{\Delta t}=96.32 \% & R=R_{\delta A} \cdot R_{\Delta t}=0.55 \times 10^{-8} \text { (for the } 11^{\text {th }} \text { series) }
\end{array}
$$

The efficiency of selection of the $10^{\text {th }}$ and $11^{\text {th }}$ series is practically identical $(\approx 96 \%)$, and the rejection factor depends on the water transparency and decreases 4 times after new water treatment.

As a whole, the rejection factor of the order $10^{-8}$ has been reached, two orders of magnitude better than in the previous studies [1, 2], due to the increase of the number of QSMs in the setup (from 27 to 91) and the refinement of the selection technique.

\subsection{Intensity of the muon flux}

By means of the above criteria, the direction for 3.84 million 'OneTrack' events was determined, among them there are 4074 albedo events with zenith angles of $91^{\circ}-95^{\circ}$. For these events, zenith and azimuth angles of the tracks were determined.

Distributions of the azimuth angle for muons coming from above (with zenith angle $\leqslant 89^{\circ}$ ), and from the bottom (with zenith angle $\geqslant 91^{\circ}$ ) are shown in figure 4 .

Distinct central peaks in figure 4 correspond to the events in which muon passes through supermodules arranged opposite to each other (pair SM07-SM00 and SM06-SM01), right and left peaks correspond to cross-direction tracks. The distribution of albedo muons is not symmetric. It is 

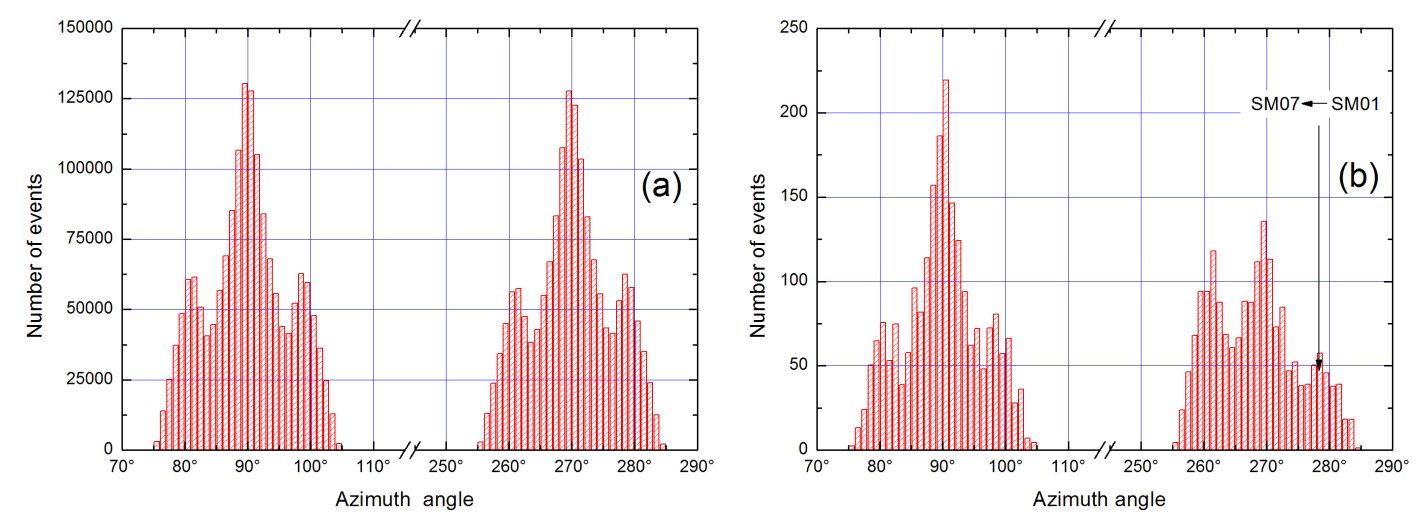

Figure 4: Azimuth angle distribution of 'OneTrack' events for zenith angles $\leqslant 89^{\circ}$ (a) and albedo event with zenith angles $\geqslant 91^{\circ}(\mathrm{b})$.

determined by the topography of the surface in the surrounding area. Muons in the range of angles $75^{\circ}-105^{\circ}$ come from the valley of the Moscow river. The lack of muons in the range of angles $255^{\circ}-285^{\circ}$ (most visible near $280^{\circ}$ ) corresponds to muons coming from the side of a hill.

The distribution of events over the measured range of zenith angles is presented in figure 5 (a). Taking into account the acceptance calculated by means of Monte-Carlo technique taking into account the structure of the coordinate detector, the intensity of muons near the horizon is calculated in the following way:

$$
I_{\mu}=\frac{N(\theta)}{S \Omega(\theta) \cdot T \cdot \eta}
$$

here:

$N(\theta)$ is the number of tracks in a given zenith angle bin;

$S \Omega(\theta)$ is the acceptance of DECOR in the same angular bin;

$T$ is 'live' time of registration;

$\eta$ is the efficiency of particle motion direction determination.

Integral muon intensity calculated with equation (2) is shown in figure 5 (b). Estimates for the two series of measurements are practically the same.
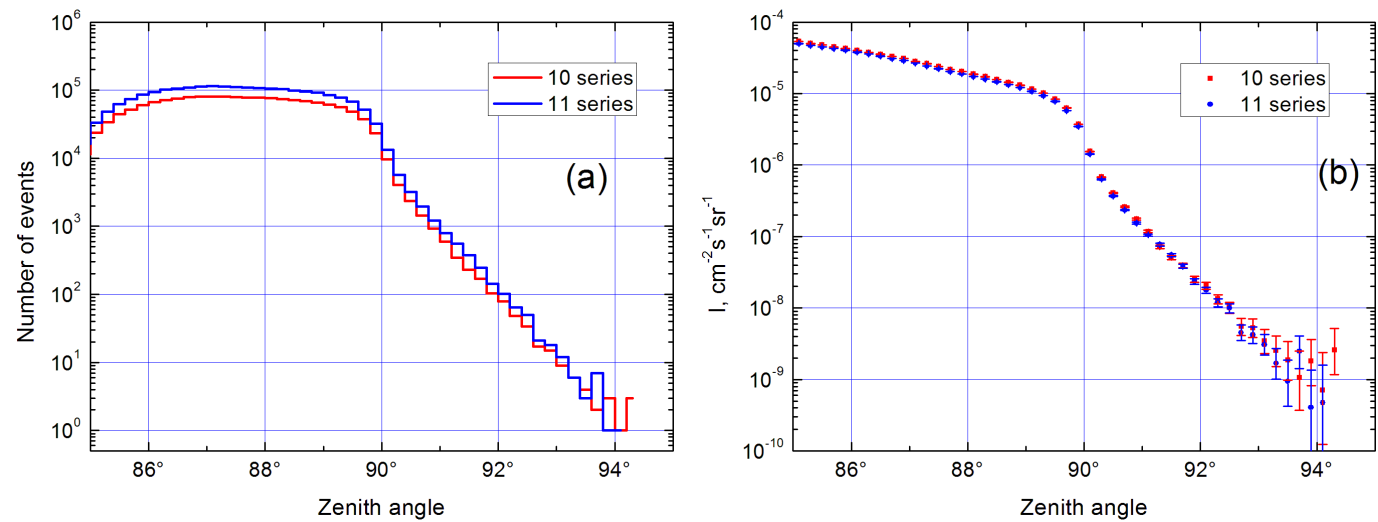

Figure 5: (a) Distribution of selected events in zenith angle estimate; (b) intensity of muon flux. 


\section{Simulation of scattering processes}

Simulation of muon penetration through a layer of ground with a flat surface by means of Monte-Carlo method has been performed. In simulations, four models are used: multiple Coulomb scattering in Gaussian approximation [6], Moliere theory [7], and model taking into account the finite size of nuclei and point-like nuclei according to [8].

Muon energy was simulated as a function of zenith angle based on differential spectrum of muons, calculated by the formulas of L.Volkova [9] and assumption about the power spectrum of primary cosmic rays with integral slope spectrum index $\gamma=1.7$.

The results of simulation are compared with experimental data in figure 6. The figure shows that the number of albedo muons for Moliere theory coincides with the results of point-like nucleus model, and with the increase of zenith angle decreases slower than in the model of finite-size nucleus. Results of calculations with Gaussian approximation are close to the dependence obtained for the model with a finite-size nucleus (with accurate nuclear form factors). The last two models significantly better describe the experimental dependence.

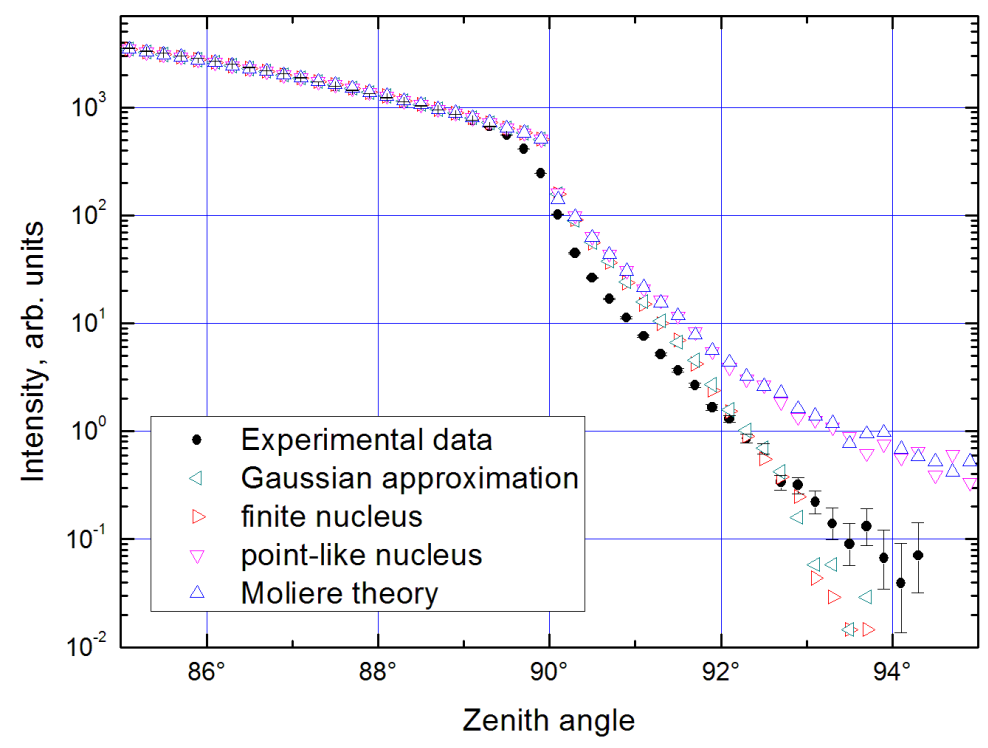

Figure 6: Comparison of experimental data and simulation results.

Figure 7 shows the distribution of simulated albedo muons in the maximum depth of penetration into the ground. The simulation is performed for the model with the finite size of the nucleus [8]. From figure 7, it follows that the albedo muon flux mainly is formed at a depth of less than $100-200 \mathrm{~g} / \mathrm{cm}^{2}$, and near the horizon $\left(90^{\circ}<\theta<91^{\circ}\right)$ a large contribution give high-energy muons, which only 'touched' the surface and then flew back.

\section{Conclusions}

Selection criteria for albedo events in the experimental complex NEVOD-DECOR on the basis of amplitude and time-of-flight techniques have been refined and tested. The intensity of muons with energies $>7 \mathrm{GeV}$ in zenith angle range $85^{\circ}-95^{\circ}$ has been measured. Simulation of muon 


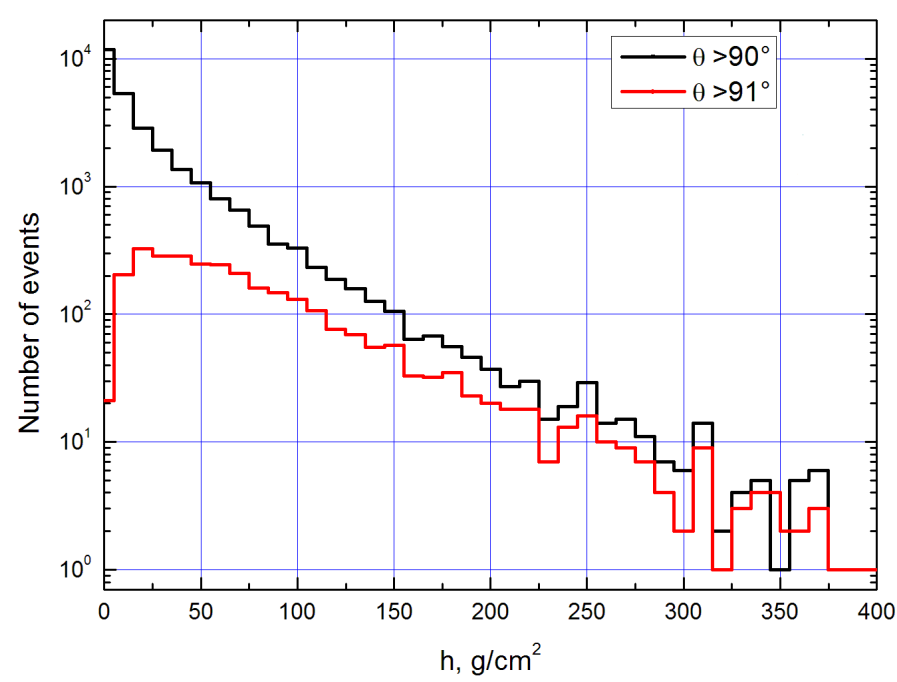

Figure 7: Distribution of simulated albedo muons in the maximum depth of penetration. Black line - all albedo muons, red line - albedo muons with zenith angle more than $91^{\circ}$.

penetration through a flat layer of ground shows that the main process of albedo muons formation in the range $91^{\circ}-94^{\circ}$ is the multiple Coulomb scattering. The calculations for the model of point-like nuclei (including the Moliere theory) lead to an overestimation of the intensity of the albedo muons.

The work has been performed at the Unique Scientific Facility NEVOD with the state support provided by the Ministry of Education and Science of the Russian Federation (project RFMEFI59114X0002, and the grant of the President of the Russian Federation MK-7145.2015.2).

\section{References}

[1] I.I. Yashin et al., Measurements of albedo muon intensity at the Earth's surface, Proc. 28th Int. Cosmic Ray Conf., Tsukuba, 3, 1195 (2003).

[2] D.A. Timashkov et al., Albedo muons: new data and calculations, Int. J. Mod. Phys., A 20, 6977 (2005).

[3] V.M. Aynutdinov et al., Neutrino water detector on the Earth's surface (NEVOD), Astrophys. Space Sci., 258, 105 (1997).

[4] N.S. Barbashina et al., A coordinate detector for studying horizontal fluxes of cosmic rays, Instrum. Exp. Tech., 43, 743 (2000).

[5] V.V. Kindin et al., Investigating the relationship between the NEVOD Cherenkov water detector's response and water transparency, Bull. Russ. Acad. Sci. Phys., 79, 421 (2015).

[6] Reviews of Particle Physics, Particle Data Group, Section 32.3 'Passage of particles through matter' (2014).

[7] H.A. Bethe, Moliere's theory of multiple scattering, Phys. Rev., 89, 1253 (1953).

[8] A.V. Butkevitch, R.P. Kokoulin, G.V. Matushko, S.P. Mikheyev, Comments on multiple scattering of high-energy muons in thick layers, NIM, A 488, 282 (2002).

[9] T.P. Amineva,V.A. Astafiev, A.Ya. Varkovitskaya, L.V. Volkova et al., Study of high energy cosmic ray muons with X-ray emulsion chambers, 'Nauka', Moscow (1975, in Russian). 